\title{
ORGANIZAÇÃO DO XXVII CONGRESSO BRASILEIRO DE ENFERMAGEM
}

\author{
H O M E N A G E M
}

Sua Excia. o Sr. Edvaldo Brandão Correia

Vice-Governador do Estado da Bahia

Sua Excia. o Sr. Carlos de Menezes Sant'Anna

Secretário de Educação

Sua Excia. o Sr. Lafayete de Azevedo Pondé

Magnífico Reitor da Universidade Federal da Bahia

Sua Excia. o Sr. Mons. Eugênio de Andrade Veiga

Magnífico Reitor da Universidade Católica do Salvador

Sua Excia. Reverendíssima Dom Avelar Brandão Vilela

Cardeal Arcebispo Primaz do Brasil

Sua Excia. o Sr. Cel. Durval de Mattos Santos

Comandante da Polícia Militar do Estado

Sua Excia. o Sr. José Ramos de Queiroz

Superintendente Regional do Instituto Nacional de Previdência Social

Sua Excia. o Sr. Carlos Alberto Sampaio Pereira

Diretor Regional Fundação Serviços de Saúde Pública

Sua Excia. o Sr. Almirante Ernesto Carneiro Ribeiro

Comandante do $2 .^{\circ}$ Distrito Naval

Sua Excia. o Sr. General Adir Fiuza de Castro

Comandante da 6. ${ }^{\mathrm{a}}$ Região Militar

Sua Excia. o Sr. Brigadeiro do Ar Osvaldo Terra Faria

Comandante do Comando Costeiro

Sua Excia. Sr. Honorato Viana de Castro

Presidente da Assembléia Legislativa do Estado

Sua Excia. o Sr. José Pires Castello Branco

Presidente da Câmara Municipal de Salvador

Sua Excia. o Sr. Plínio Mariani Guerreiro

Presidente do Tribunal de Justiça

\section{CONVIDADOS ESPECIAIS}

Sra. Maria Amélia Menezes Santos

Sra. Maria Lúcia Hage 


\section{COLABORADORES}

Governo do Estado da Bahia

Prefeitura Municipal de Salvador

Secretaria de Saúde do Estado

Secretaria de Educação e Cultura do

Estado

Secretaria do Trabalho e Bem-Estar Social

Fundação Serviços de Saúde Pública

Serviços Médicos - Cirúrgicos da Bahia

Universidade Federal da Bahia

Universidade Católica do Salvador

Polícia Militar do Salvador

Associação de Hospitais do Estado da

Bahia

Faculdade de Enfermagem - U.C.S.

Escola de Enfermagem - U.F.Ba.

Escola de Auxiliares de Enfermagem Rosa Gattorno

Banco Real

Banco do Estado da Bahia

Banco Econômico S.A.

Banco do Nordeste S.A.

Banco Brasileiro de Descontos

Banco de Desenvolvimento do Estado da Bahia

Cremer S.A. - Produtos Têxteis e

Cirúrgicos

Darrow Laboratórios S.A.

Johnson \& Johnson - Divisão Hospitalar

e Ethicon

Laboratório Lepetit

Laboratório Schering

Leboratório Valmont - Winthrop

Laboratório Ciba - Geigy Química S.A.

Laboratório Usafarma

Laborterápica Bristol

Laboratório Abott

Laboratório Upjohn - Produtos Farmacêuticos Ltda.

Laboratório Lafi

Laboratório Berlimed

Indústria de Papéis Simão S.A.

Indústria York S.A.

Federação da Indústria e Comércio

Companhia Industrial e Comercial

Brasileira de Produtos Alimentares Nestlé

Água Mineral Dias D'Avila

Bahia Frutos S.A.
Maguary S.A.

Cia. Antártica do Nordeste

Indústria Chardler

Coca-Cola

Sanave S.A. Nacional de Veículos

Óticas Oliveira

Paes Mendonça S.A.

Antonio Carlos Magalhães

Clériston Andrade

Ênio Faria Rozendo Pinto

Sibra Eletrosiderúrgica Brasileira S.A.

Procilab - Produtos de Cirurgia e

Laboratórios Ltda.

Sociedade Luso Brasileira

Nunes Tecidos

Restaurante Moenda

Empresa de ônibus Camarogipe

Empresa de ônibus Bomfim

Raul Seixas Comp. Ltda.

Dr. Wilton Nascimento - Gerente da P.O.S.E.

Viação Aérea São Paulo - Vasp

Telecomunicações da Bahia S.A.

Empresa Brasileira de Correios e

Telégrafos

Departamento Estadual de Trânsito

Associação Atlética da Bahia

Irte - Instituto de Reabilitação Terapêutica e Estética

Instituto Bahiano de Oftalmologia e Prevenção da Cegueira

Hospital de Doenças do Tórax.

Bahiatursa

TV Aratu - Canal 4

TV - Itapoan - Canal 5

Jornal da Bahia

A Tarde

Tribuna da Bahia

Diário de Notícias

Jornal do I.C.

Jornal "O Macon"

Profa. Maria José Oliveira

Prof. Laert Pedreira Neves

Maurício Nonato - Costureiro

Gráfica Fontes

Dr. Hernani Gutierres

Hospital Prof. Edgard Santos

Hospital Ana Nery - INPS

Cirúrgica Fernandes S.A. 
Comissão de Coordenação

- Ma. da Graça Simões Côrte Imperial - Presidente

- Dra. Amália Corrêa Carvalho - 2. ${ }^{\mathrm{a}}$ Vice-Presidente

- Taka Oguisso - Tesoureira

- Clarice Ferrarini - Secretária Executiva

Comissão de Temas

- Dra. Maria Ivete Ribeiro de Oliveira - Presidente

- Eurídes Correia Rocha

- Maria Hélia de Almeida

- Clarice Oliveira

Comissão de Recomendações

- Lígia Paim

- Maria Camargo de Oliveira Falcão

- Celina de Arruda Camargo

Comissão Executiva

- Jandyra Orrico - Presidente

- Eunice Marinho Alcântara - Tesoureira

- Edelita Coelho de Araújo - Secretária

- Gicele Dórea

- Nair Fábio da Silva

- Clara Wolfovich

- Carmélia Sarno Neves

- Iraídes Teixeira de Carvalho Andrade

- Creuza de Souza Silva

\section{SUB COMISSÕES}

Secretaria

- Glacy Vieira Jasmim - Coordenadora

- Creuza de Souza Silva

- Zuleika Actis

- Tânia Márcia Bueno Baraúna

- Maria Agda Mendonça Lavingne

- Maria Ester Pinto de Abreu

Finanças

- Eunice Marinho Alcântara - Coordenadora

- Heleno Amaro da Silva

- Maria Myrtes Araújo Magalhães

- Rute Guedes de Souza

Registro

- Maria José Arleo Barbosa Amorim - Coordenadora

- Ieda Helena Hurst

- Leny Rocha Andrade

- Delvair de Brito Alves 
- Edeltrudes do Espírito Santo Alves

- Maria de La Purificacion Garcia Herranz

- Maria Clayde Teixeira Barroso de Oliveira

- Maria da Graça Vila Serra Gomes

- Célia Vila Serra

- Vilma Alves Cabral Carvalho

- Celeste Maria Mendes Bezerra

- Elza Maria da Silva Aragão

- Inea Pinheiro Santana da Silva

- Heloniza Oliveira Gonçalves Costa

- Noélia Conceição Oliveira

- Mara de Araújo Almeida

- Rosamaria Silva Nunes

- Maria D’Ajuda Melo Guimarães

- Neusa Chaves Pedreira

Hospedagem

- Maria Jenny Silva Araújo - Coordenadora

- Aidil de Jesus Alcoeres

- Edith de Figueiredo Domingues

- Jurene Brito Ferreira

- Cecília Maria Costa Souza

- Nicelita Maria Santos Guimarães

- Carlinda Maria Santos Guimarães

- Antonia Alves de Oliveira - Ir.

Social

- Anaíta de Oliveira Costa - Coordenadora

- Alayde Vieira de Roman

- Ednelza Soares Feitosa

- Maria Roseilda Bispos

- Stela Sampaio Galvão

- Celuta Pedreira Costa

- Maria Olinda Silva

- Helienar Helena da Silva

- Josicelia Fernandes

- Maria Alice Saraiva Nogueira - Ir.

Monitoria

- María das Dores Silva - Coordenadora

- Vera Lúcia Souza Bastos - Coordenadora

- Ileana Marlene Veloso Falcão

- Maria do Carmo Ramos

- Rosa Lúcia Rodrigues Alves

Divulgação

- Iraides Teixeira de C. Andrade - Coordenadora

- Daisy Schwab Rodrigues

- Maria Angélica Valverde Martins

- Valdete Santos Botelho 
Recepção

- Evanilde Alencar Carvalho - Coordenadora

- Enede Andrade Cruz

- Alba Guedeville

- Nilzete Gomes Massa

- Silvia Berbet Hagge

- Eny Alves Braga Duran

- Ailda Cunha da Motta Gesteira

- Valdivina Andrade

- Maria Neuza de Melo

- Maria Nelsonita Carneiro de Lima

- Hisako Uchida de Freitas

- Marinalda Freitas Santa Cruz

Local

- Sílvia Dias Pimentel dos Santos - Coordenadora

- Nilcéia Nascimento

- Floripes Cavalcante Farias

- Célia Coelho Dantas

- Remilda Silva Santos

- Ana Célia Queiroz Bastos

- Vera Márcia Leal

- Sônia da Silveira e Souza

- Lídia Gomes

- Marienne Passos

- Maria Jesia Vieira

\title{
SESSÃO DE INSTALAÇÃO
}

\author{
27 de julho de 1975 \\ 20:30 horas \\ Teatro Castro Alves
}

Abertura da Sessão

Hino Nacional

Saudação aos Congressistas

Enf. Jandyra Orrico - Presidente da Associação Brasileira de Enfermagem Seção da Bahia

Discurso da Presidente da Associação Brasileira de Enf€rmagem

Enf. Maria da Graça Côrte Imperial

Outorga do Título de Sócia Honorária A

Dra. Maria Ivete Ribeiro de Oliveira

Apresentação

Dr.a Amália Corrêa de Carvalho

Encerramento

Apresentação do Grupo de Dança da Universidade Federal da Bahia 


\section{TEMAS OFICIAIS}

\section{Tema I}

Integração do ensino e serviço de enfermagem

1. ${ }^{\mathrm{a}}$ Sessão Plenária

Dia 28/07/75 — segunda-feira

14 às 18 horas - Teatro Castro Alves

Presidente

Elvira De Felice e Souza - UFRJ

Relatores

Cícero Adolfo da Silva - UFBa.

Maria Ivete Ribeiro de Oliveira - UFBa

Edla Dalva Moreira. - INPS - RJ

Hyeda Rigaud Castro - SP

Maria Isabel Santos - UFPe.

Tema II

Participação de pessoal ce Enfermagem nos programas de assistência à saúde nas populaçeõs da zona rural

2. ${ }^{a}$ Sessão Plenária

Dia 29/07/75 — Terça-feira

08 às 12 horas - Teatro Castro Alves

Presidente

Harley Padilha — Ser. Saúde - Bahia

Relatores

Judith Feitosa de Carvalho

Lasthemia Miranda Chiari - PA.

Tema III

Problemas de Assistência de Enfermagem nos Hospitais e Clínicas particulares de grandes centros urbanos

3. ${ }^{a}$ Sessão Plenária

Dia 30/07/75 - Quarta-feira

08 às 12 horas - Teatro Castro Alves

Presidente

Emerson Spinola Ferreira - UFBa.

Relatores

Taka Oguisso - USP

Inéa Pinheiro Santana Silva - BA.

Vilma Carvalho - UFRJ.

Tema IV

Recentes pesquisas em enfermagem. 
4. ${ }^{\text {a }}$ Sessão Plenária

Dia 31/07/75 - Quinta-feira

08 às 12 horas - Colégio das Sacramentinas.

ÁREA I

Assistência de Enfermagem ao adulto e ao velho.

Presidente

Francisca Nogueira Soares - SP.

1 - "Descrição das manifestações do comportamento apresentado por pacientes com diagnóstico de Esquizofrenia"”'.

Relatora

Maguida Costa Stefaneli - USP

2 - Indicações e finalidades do cateterismo venoso central em Unidades de Terapia Intensiva - SP.

Relatora

João Augusto Filho

3 - Complicações do cateterismo venoso central em pacientes internados em Unidades de Terapia Intensiva.

Relatora

Simone Santos Robim

4 - Estudo comparatório da dosagem do ácido lático no sangue arterial, venoso-central e periférico em doentes, de Unidades de Terapia Intensiva.

Relatora

Gelse Mary Zerbeto - SP.

5 - Estudo exploratório da consulta de enfermagem.

Relatora

Ieda Barreiro Castro

6 - Avaliação de degermação das mãos com hexaclorofeno, compostos quaternários de amônio e sabão comum.

Relatora

Ana Maria Palermo Cunha - USP - Sp.

7 - A aplicação de um regime de solicitações no período pós-operatório em pacientes submetidos a cirurgia abdominais.

Relatora

Lia Hoelz Alvares 
8 - Informação dos problemas de saúde recebidos pelos pacientes internados.

Relatora

Tokico M. Morise.

ÁREA II

Assistência à mãe e/ou à criança.

Presidente

Hyeda Rigaud Castro - SP

1 - Escoamento de sangue fonicular como método auxiliar no descolamento da placenta.

Relatora

Maria Zofia Schubert

2 - Influência dos exercícios respiratórios e do estado civil no comportamento de parturientes.

Relatora

- Wanda Escobar da Silva Freddi - USP

3 - Observação clínica das ocorrências no processo de mumificação, queda do coto e cicatrização da ferida umbilical em recém-nascidos submetidos a dois cuidados de higiene.

Relatora

Dulce Maria de Freitas - USP - RP.

4 - Papel da enfermeira junto às mães de crianças hospitalizadas.

Relatora

Ester Moraes - USP.

5 - "Pesquisa de suspensão de cirurgia e respectivas relações causais".

Relatora

Doralice P. Noronha

AREA III

Assistência de enfermagem à família e/ou à comunidade.

Presidente

Josefina Melo - AM

1 - "Algumas medidas sanitárias utilizadas por familias que demandam os Serviços dos Centros de Saúde em Salvador".

Relatora

Ruth Guedes Souza - UF'Ba. 
2 - "Vacinação B. C. G. via intradérmica no Brasil - UFRJ.

Relatora

Ieda Barreira

ÁREA IV

Educação em enfermagem.

Presidente

Padre Ivo Gelain - SP.

1 - "Dificuldades dos estudantes de enfermagem frente aos doentes nentais internados".

Relatora

Evalda Cançado Arantes - USP.

2 - "O significado psicológico də, dor para docentes e estudantes de enfermagem e docentes de psicologia".

Relatora

Wanda Horta - USP.

3 - "O significado psicológico de palavras relacionadas à saúde, para grupos de estudantes universitários cuja formação visa o ser humano Enfermagem e Psicologia".

Relatoras

Wanda Horta - USP.

4 - "Formação do enfermeiro a partir de técnicas de desempenho de papel uma experiência em enfermagem médico-cirúrgica".

Relatora

Zilma Ramos de Oliveira - USP - RP.

5 - "Atuação da Enfermagem de campus avançado".

Relatora

Maria José da Silva - MG.

OBS.: Os trabalhos inscritos, cujos resumos não foram enviados à comissão em tempo hábil deixam de figurar nesta programação.

\section{TEMAS LIVRES}

AREA I

Organização e Administração de Serviços de Enfermagem

Dia 29/07/75 - Terça-feira

14 às 18 horas - Colégio Sacramentinas. 
Presidente

Dawiame Dayse Rieker - AM.

Trabalhos:

1 - "Organização do Serviço de Enfermagem em hospital particular - Implaníação de sistemas de controle".

Relatora

Maria Lúcia Pimentel de Assis Moura - SP.

2 - "Treinamento em Serviço".

Relator

Rangel Teixeira Salgado - MG.

3 - Experiência de enfermagem em três unidades descentralizadas no município de Londrina - Paraná.

Relatora

Kiko Kaminari Shibayama - PR.

4 - "Papel da enfermeira na aquisição de material técnico".

Relatora

Vitória secaf - SP.

5 - "Enfermagem no Hospital Juliano Moreira passado e presente".

Relatora

Maria de la Purificacion Garcia Herranz

6 - Visita Hospitalar: Aspectos Humanitários, Social e Administrativo.

Relatora

Raim!nnda Costa Lima - USP - SP.

ÁREA II

Saúde Pública

Dia 29/07/75 - Terça-feira

14 às 18 horas - Colégio Sacramentinas.

Presidente

Adalgisa Vieira Matos - RJ.

Trabalhos:

1 - "Preparo de retaguarda para atendimento de meningite, numa pequena comunidade".

Relatora

Tokiko Murakana Moriya - RP - SP.

2 - "Atividades da Curiosa na Cidade de Salvador".

Relatora

Iracy Silva Costa - BA

3 - Atuação do Serviço de Enfermagem do Hospital das Clínicas da F. M. U. S. P. durante a epidemia de meningite do ano de 1974 . 
Relatora

Maria Lúcia Toledo Vasco - USP.

ÁREA III

Educação èm Enfermagem

Dia 29/07/75 - Terça-feíra

14 às 18 horas - Colégio Sacramentinas.

Presidente

Eloita Pereira Neves - UFSC.

Trabalhos:

1 - "Como vimos o curso de especialização em enfermagem médico-cirúrgico sob a forma de residência".

Relatora

Delvair de Brito Alves - BA.

2 - "Curso supletivo de qualificação para auxiliar de enfermagem".

Relatora

Francisca Alves de Souza - PE.

3 - "Considerações gerais sobre o início de carreira".

Relatora

Paola Maresca - SP.

4 - Abordagens novas na atenção de enfermagem e a necessidade de comunicação entre os enfermeiros dos serviços e do ensino.

Relatora

Haidée Guanais Dourado - RJ.

5 - Consulta de Enfermagem

Relatora

Wanda Aguiar Horta - USP.

6 - Implantação da A. B. E. E.

Relatora

Francisca Nogueira Santos

ÁREA IV

Assistência de Enfermagem

Dia 29/07/75 - Terça-feira

14 às 18 horas - Colégio Sacramentinas.

Presidentes

Maria Camargo Falcão

Maria de Lourdes Goes Miller 
Trabalhos:

1 - "Pacientes hospitalizados - responsabilidade da enfermeira na prevenção de limitações físicas".

Relatora

Marlúcia Nunes Comarú - SP.

2 - "A sociedade e a rejeição ao doente mental".

Relatora

Júlia Nogueira Brandão Ayres - BA.

3 - "Ação da temperatura de ebulição da água acrescida de sal, de ácido ou de base sobre as bactérias".

Relatora

Tokiko Mirakana Miriya - Ribeirão Preto - SP.

4 - "O papel da enfermeira na assistência à gestante sadia".

Relatora

Léa Cecília Musfeldt - UFRGS.

5 - "Assistência de enfermagem às gestantes de alto risco".

Relatora

Adeline Maria Costa - MG.

6 - "Assistência de Enfermagem em Berçários".

Relatora

Maria Lúcia Pimentel de Assis Moura - SP.

7 - "Cuidados de Enfermagem nos pacientes portadores de "shunt" e fístula artério venosa".

Relatora

Marizete Pereira da Silva

8 - "Assistência de Enfermagem a paciente com desordens circulatórias cerebrais".

Relatora

Maria Lúcìa C. Robazzi - USP - RP.

9 - "Atuação a enfermeira junto aos doentes com distúrbios do metabolismo hidrossalino".

Relatora

Clarice Oliveira - UFBa.

10 - "Problemática Bio-Psico-Social de pacientes portadores de úlcera da perna'.

Relatora

Edna Maria Teixeira de Carvalho - SP.

11 - O papel da enfermagem na interpretação dos valores da osmolalidade plasmática e urinária em pacientes admitidos em Unidade de Terapia Intensiva. 
Relatora

Simone Santos Robim

12 "Assistência de Enfermagem em Moléstias Transmissíveis'.

Relatora

Edoilia Maia Teixeira - USP - SP.

13 - Implantação de novos métodos de trabalhos para assistência ao paciente de Hanseniase na Clínica Dermatológica".

Relatora

Tomie Mori - USP - SP.

14 - "Cuidados de Enfermagem a pacientes portadores da prótese peritoneal de Deane".

Relatora

Ester Ferreira da Silva - USP - SP.

15 - "Assistência de Enfermagem em Pacientes portadores de miastenia gravis".

Relatora

Maria Dulce Cardoso - USP - SP.

16 - "Assistência de Enfermagem ao paciente com fotossensibilização".

Relatora

Laurinda Madureira Gandolla - USP - SP.

17 - "Contribuição de Enfermagem no diagnóstico e pesquisa médica na dermatite de contato".

Relatora

Aurora Cordeiro - USP - SP.

18 - "Balneoterapia do paciente queimado, sistematização do atendimento de enfermagern".

Relatora

Maria de Lourdes Croes Muller - USP - SP.

19 - "Aspectos de Enfermagem na execução da hemodiálise a domicílio uma experiência”.

Relatora

Antonia da Graça Silva - USP - SP.

20 - "Unha - fator de contaminação".

Relatora

Luci Romero Grupiom - USP - SP.

21 — Tetânicos - Assistência de Enfermagem"”.

Relatora

Maria Rita Silva.

22 - "Assistência de Enfermagem em Moléstias Transmissíveis, contribuição para o tratado de Doenças Infecciosas e Parasitárias". 


\section{PROGRAMA DE ATUALIZAÇÃO}

Dia 30/07 e 31/07/75 - Quarta-feira

14 às 18 horas.

1 - Metodologia Científica

Professora: Célia Maria Leal Braga (UFBa)

Sala 1

2 - Atualização em enfermagem psiquiátrica

Professora: Tereza Sena (UFRJ)

Sala 2

3 - Papel do enfermeiro nas'alterações metabólicas

Professora: Clarice Oliveira (UFBa)

Auđitório

4. - Planejamento de Saúde

Professores: Judith Feitosa Carvalho

Lasthemia Chiari

Sala 4

5 - Atualização em temas ortopédicos e traumatclógicos Professora: Neide Goes Benuth (USP)

Sala 4

6 - Humanismo e anti-humanismo em conflito Professor: Pedro Dalle Nogare (UFBa)

Sala 5

Dia $01 / 08 / 75$

7 - Legislação em enfermagem

Professora: Maria Rosa de Souza Pinheiro (USP)

Sala 1

8 - Assistência ao Prematuro

Professora: Célia Coelho Dantas (UFBa)

9 - Atualização em Centro Cirúrgico

Professora: Maria Falcão (USP)

10 - Novos aspectos da enfermagem comunitária

Professoras: Stella Barros

Adalgisa Vieira

Sala 4 